\title{
EXPERIMENT FOR INTEGRATING DUTCH 3D SPATIAL PLANNING AND BIM FOR CHECKING BUILDING PERMITS
}

\author{
L. van Berlo ${ }^{\mathrm{a}}$ T. Dijkmans ${ }^{\mathrm{a}}$, J. Stoter ${ }^{\mathrm{b}}$ \\ ${ }^{a}$ Netherlands organisation for applied scientific research TNO, PO Box 49, 2600 AA, Delft, The Netherlands - \\ (leon.vanberlo, tim.dijkmans)@tno.nl \\ ${ }^{\mathrm{b}}$ Delft University of Technology, Delft, The Netherlands - j.e.stoter@tudelft.nl
}

Commission II, WG II/2

KEY WORDS: BIM, Information Modelling, 3D data integration, City and Urban planning, Standards for geospatial technologies

\begin{abstract}
:
This paper presents a research project in The Netherlands in which several SMEs collaborated to create a 3D model of the National spatial planning information. This 2D information system described in the IMRO data standard holds implicit 3D information that can be used to generate an explicit 3D model. The project realized a proof of concept to generate a 3D spatial planning model. The team used the model to integrate it with several 3D Building Information Models (BIMs) described in the open data standard Industry Foundation Classes (IFC). Goal of the project was (1) to generate a 3D BIM model from spatial planning information to be used by the architect during the early design phase, and (2) allow 3D checking of building permits. The team used several technologies like CityGML, BIM clash detection and GeoBIM to explore the potential of this innovation. Within the project a showcase was created with a part of the spatial plan from the city of The Hague. Several BIM models were integrated in the 3D spatial plan of this area. A workflow has been described that demonstrates the benefits of collaboration between the spatial domain and the AEC industry in 3D. The research results in a showcase with conclusions and considerations for both national and international practice.
\end{abstract}

\section{INTRODUCTION}

The benefits of aligning the domains of Building Information Models (with data encoded in IFC) and 3D geo-information (with data encoded in (City)GML) are widely recognized in both 3D data communities. Geo-information provides a framework for BIM data. On the other hand BIM data can be an important source for 3D geo-information. Sometimes the two domains even model the same world objects such as buildings, bridges and infrastructure. However, since the two domains have different perspectives resulting in different modelling approaches, the alignment of the two domains is not straightforward and requires still a lot of research.

Such research should acknowledge the differences between both types of data. Differences exist in the extent of areas of interest. GIS is characterised by coverage of large areas (e.g. a complete city) and lower precision, while BIM is characterised by its local and very detailed approach necessary for reliable structural calculations. Also the modelling approaches of CityGML and IFC differ, i.e. IFC contains much more classes and also allows non-hierarchal relationships, where CityGML contains a limited number of classes structured via hierarchical relationships.

Several studies have shown that a conversion between IFC and CityGML is possible and that such conversion allows integration of both types of data, see for example ((Isikdag and Zlatanova 2009) (van Berlo and de Laat 2011) (El-Mekawy, Östman, and Shahzad 2011) (Bormann 2010). Still many issues remain unresolved.

This paper presents a case study that shows how 3D data from the geo-information domain, i.e. 3D spatial plans and environmental data, can be integrated with 3D designs, modelled in IFC, to support the design and construction process considerably. The case study realized a proof of concept of a technical environment for architects that allows 3D checking of building designs against 3D spatial planning objects in the design process. These 3D spatial planning objects describe building regulations and environmental data in 3D. In a later stage the integrated data can be used to validate the 3D design against the regulations to check whether a building permit can be issued.

The aim of the case study, carried out within a consortium of several SMEs, was to show the potential of integrating 3D geoinformation with IFC data and to provide a showcase to push better exploitation of this integration.

Other researches have studied the integration of BIM/IFC data and plan data to automatically check a "digital" building application for its consistency with legal regulations, see Benner, Geiger, and Häfele (2010). They imported the IFC data of the designed construction in the geo-information (i.e. plan) environment. The main difference with our approach is that we also import the plan information in the design (i.e. BIM) environment so that the plan information can be taken into account during the design.

\section{METHODOLOGY}

The aim of the project was to realise a proof of concept of checking building designs against building regulations laid down in spatial plans. The main steps to realise this POC were: 1) extend the $2 \mathrm{D}$ spatial planning map in $3 \mathrm{D}, 2$ ) integrate the spatial planning data with IFC designs 3) apply spatial operations to check the designs against spatial planning regulations.

\subsection{Extend 2D spatial planning map in 3D}

Spatial plans in the Netherlands are defined according to the IMRO standard (Information Model Ruimtelijke Ordening, 
Information Model Spatial Planning). This standard covers both (verbal) regulations and a spatial planning map consisting of 2D planning objects on which "allowed destinations" are defined (see Figure 1). These planning objects are mostly established by local government and in total they cover the complete area of the Netherlands. The planning objects can be queried via a national portal "http://www.ruimtelijkeplannen.nl" hosted by the Dutch Kadaster.

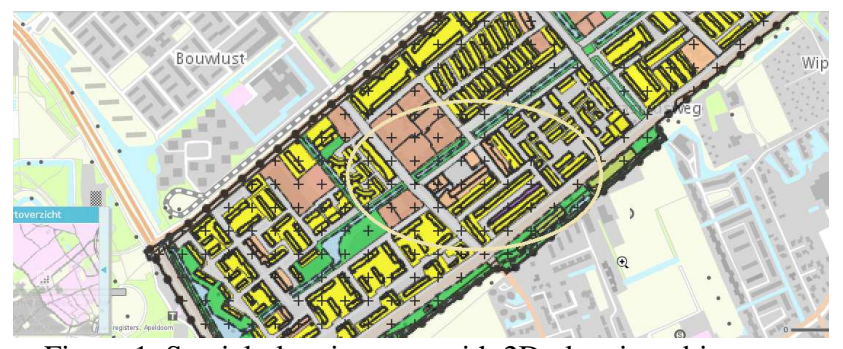

Figure 1. Spatial planning map with 2D planning objects as viewable on "http://www.ruimtelijkeplannen.nl"

Although the spatial planning maps are only available in 2D, the regulations do in fact have $3 \mathrm{D}$ components, for example the maximum allowed building height is an attribute of the $2 \mathrm{D}$ planning object.

To give these $3 \mathrm{D}$ related regulations a spatial extent in $3 \mathrm{D}$, the 2D planning objects were converted into 3D planning objects by using the maximum allowed building heights given in the regulations from the ground level (see Figure 2). This is done with a custom written computer program that transforms GML data in EPSG:28992 (the Dutch 2D national coordinate system) to EPSG:7415 (the same Dutch coordinate system, but now in 3D with height coordinates according to the Dutch NAP). During the transformation the 'maximum allowed height' attribute from IMRO is used to define the height of the object.

Result of the transformation was a GML file we called 3DIMRO GML. In this project we had issues trying to view the 3D IMRO GML model because most viewers don't support a generic 3D GML view. The solution that was used in this project was to generate a CityGML file from the 3D IMRO GML in which all the IMRO objects were modelled as CityGML Buildings. This is not the correct way to model, but gave us the opportunity to use several CityGML viewers to present the 3D IMRO model.

Our viewer of choice in this project was Sketchup. Using a plugin to import/export CityGML in and from Sketchup we were able to get the 3D IMRO data into Sketchup. Combining it with aerial photography added value to the presentation of the model.

After finding this technical data flow (2D IMRO to 3D IMRO to CityGML to Sketchup) we continued to look for more integration between 2D IMRO and the 3D presentation. One of the most visual integrations was the integration of the IMRO colour standard in 3D. The space destinations in 2D IMRO are defined in a colour schema. For example roads are grey, green is green and housing is yellow. Getting the spatial destination attribute from a 2D object, linking it to the colour schema, gives us the ability to add a colour attribute to the 3D objects. This colour attribute remains available in the 3D IMRO GML model and is also shown in Sketchup. From this moment the model was not valid CityGML anymore, but a 3D IMRO GML. There were some issues where Sketchup does not support specific colours and replaces it with a 'nearby' colour in the spectrum.

A 3D spatial plan encoded in 3D IMRO GML allows for environmental data from other sources to be added to the plan as well. In this case study for instance, data on the Dutch road network from the national portal for geo-information "http://www.nationaalgeoregister.nl" was used to model noise values as $3 \mathrm{D}$ objects.

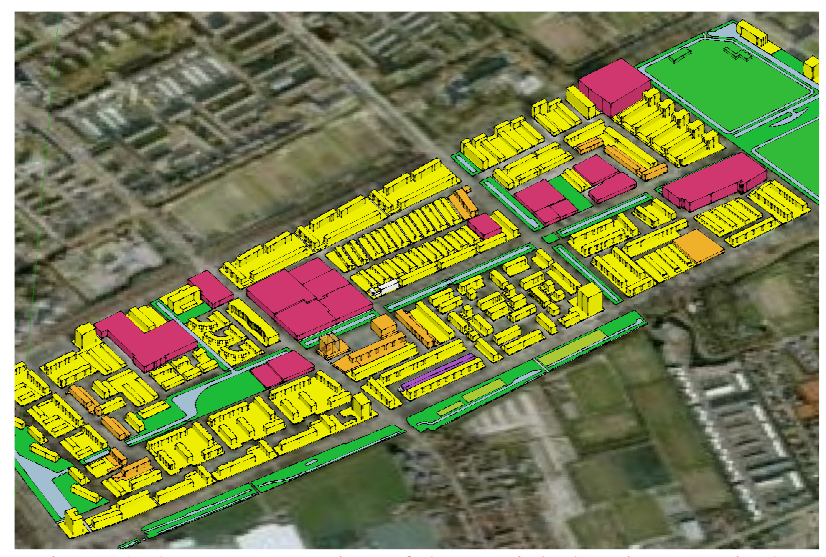

Figure 2. 3D representation of the spatial planning map in 3D IMRO GML, using attributes from the regulations such as colour and maximum allowed building height

\subsection{Integrate 3D Spatial plans with IFC data}

The integration between building designs and 3D planning objects was realised by converting the 3D spatial planning objects as IFC and import these into BIM software (see Figure 3). At first we tried to convert the 3D IMRO GML model to IFC using the FZK viewer. We found several issues with this approach like data loss in the resulting IFC, loss of the colour attribute in IFC and faces with wrongly positioned normal vectors in IFC. This is understandable because FZK viewer assumes valid CityGML and the 3D IMRO GML is CityGML with specific IMRO attributes. Parallel to the FZK viewer we custom built software to transform the base 3D IMRO GML model to IFC to tackle those issues. The result was an IFC dataset including IMRO attributes, ID's and colour. The showcase area in The Hague presented as IFC, viewed using Solibri Model viewer is shown in Figure 3.

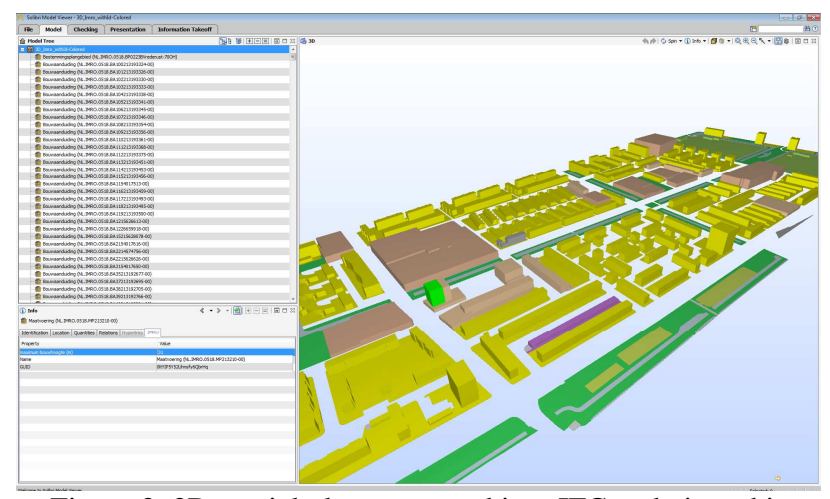

Figure 3. 3D spatial plan converted into IFC and viewed in Solibri Model Viewer

\subsection{Check the IFC designs against spatial planning regulations}

The checking of the designs is done by performing spatial analyses on the integrated data within the BIM environment.

Several types of regulation were checked in this case study: the maximum allowed building volume, the maximum percentage of build area on the site, the maximum allowed noise value on 
the façade of buildings and if protected cultural heritage in the underground is not harmed by the designs.

To automate the checking of the maximum allowed building volume and percentage of build area on the site, attributes from 2D IMRO were maintained in the 3D IMRO GML model and therefore also appear as an IFC attribute in the BIM model. This is shown in Figure 5.

These attributes make it possible to check the building design against the 3D IMRO BIM using model checking software like Solibri Model Checker. These rule checking and clash detection functionalities are standard features in BIM and therefore familiar to BIM users in the construction industry. If the model check shows that the design does not comply with regulations, it can be adjusted and the checking is performed again. The end result is a design of which the designer can be confident that it complies with the regulations in the 3D spatial plan, because the model is checked in 3D before submission for a building permit. The design can then be uploaded to the building permit portal in the IFC format. In The Netherlands, this portal currently already accepts IFC files.

\section{SHOWCASE}

The primary result of the project is a showcase that demonstrates the advantages of checking building designs against spatial planning regulations in $3 \mathrm{D}$. In this particular showcase the architect or designer of the building is the lead beneficiary of the proposed workflow. The workflow consists of 5 main steps, with a varying number of sub steps, depending on the types of regulation that are in force on a particular location.

\subsection{Workflow steps}

Step 1: The architect/designer visits the 3D spatial plan portal and selects the area of interest

The first step in the workflow will be the same as it is (or should be) in current practice, the architect looks up the spatial plan online (see Figure 4).

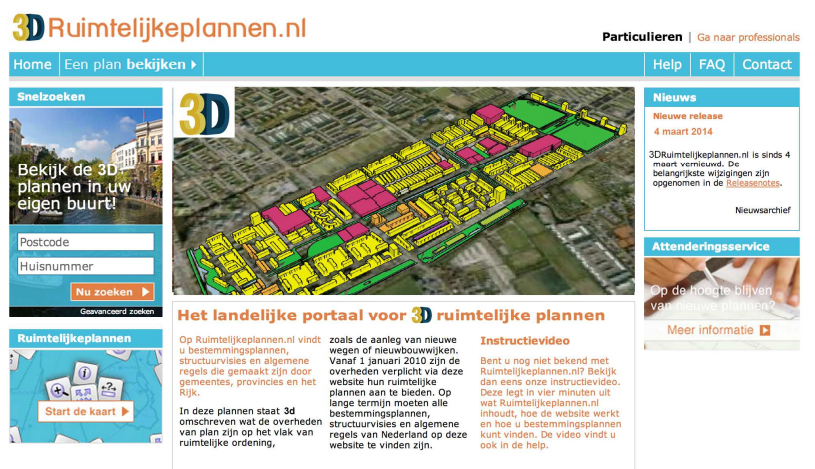

Figure 4. Mock-up of a fictional website for downloading 3D spatial plans (based on "http://www.ruimtelijkeplannen.nl")

Step 2: The architect downloads 3D spatial objects as BIM objects (IFC)

The architect can download the desired 3D spatial objects as BIM objects in IFC format. The attributes of the downloaded objects are also to be retained in the conversion from 3D IMRO GML to IFC (see Figure 5). In addition, environmental data described in $3 \mathrm{D}$ are also converted to BIM objects to allow for checking in the BIM software.

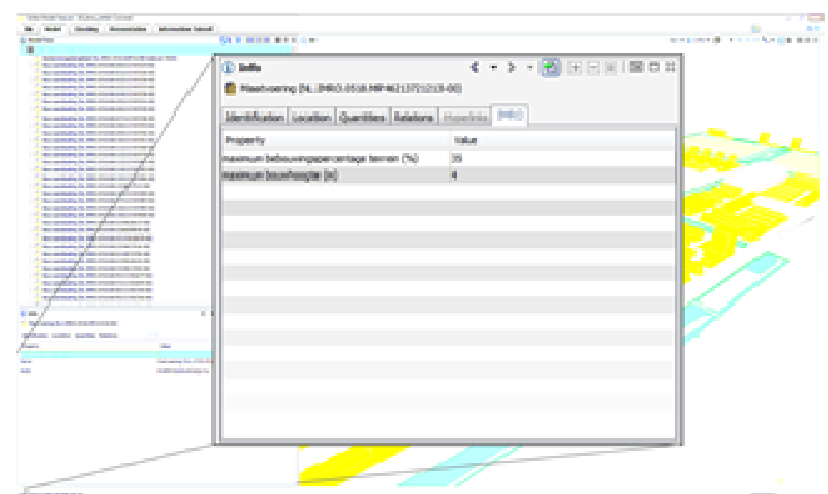

Figure 5. Attributes of the spatial objects are retained in the conversion from geo-information to BIM

Step 3: The architect uses these data as input in the BIM design software and designs the building accordingly (in BIM)

During the design process the architect can enable and disable visibility of the spatial planning regulations and design the required building accordingly.

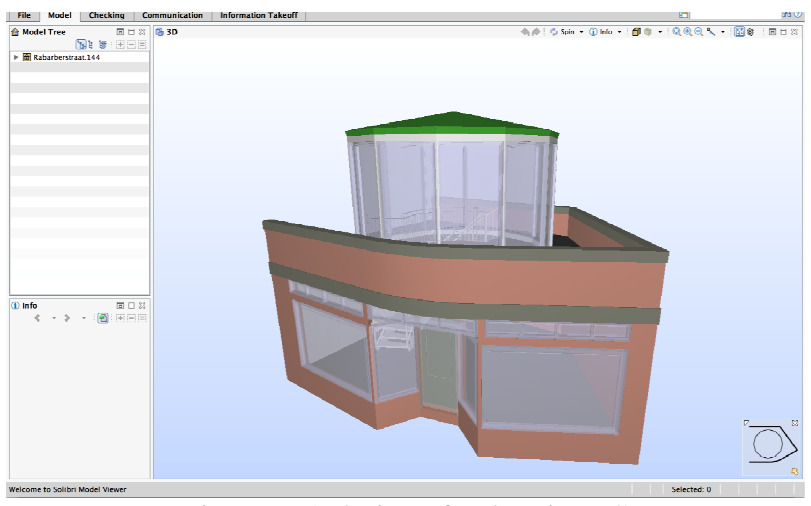

Figure 6. A design of a shop in IFC

Step 4a: The architect checks the design for regulations regarding geometry

After or (preferably) during the design process, the architect can check for the variety of regulations that is in force on the particular location that has been assigned for the building. The first type that is checked in this showcase is regulation regarding geometry. Attributes regarding maximum allowed length, width and height are assigned to the spatial object and visually represented in 3D. But regulation regarding to the maximum allowed percentage of the ground that is allowed for construction is determined by the attributes of the spatial objects and can therefore be checked.

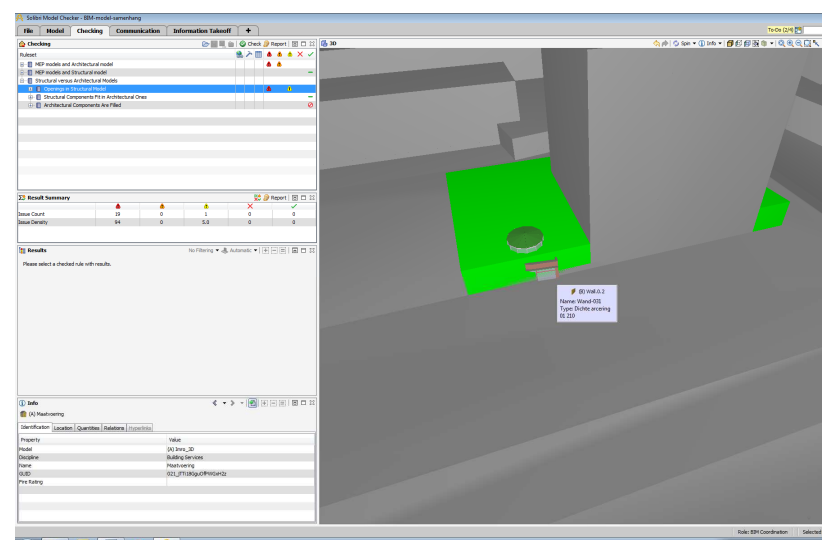

Figure 7. Model checking in IFC on spatial regulations 
Step 4b: The architect checks the design for regulations regarding noise values

Noise values in the environment as a result of road traffic, air traffic or other sources are represented in $3 \mathrm{D}$, in a similar way to the $2 \mathrm{D}$ isobel maps that are commonly used for displaying sound pressure levels in a geographic location. The third dimension grants the designer the possibility to design in a smarter way. Sound is a 3D phenomenon by definition, in traditional practice it is not treated as such. Sound pressure levels are usually displayed in $2 \mathrm{D}$, limiting the designer to the sound pressure level measured at the ground level, no matter at what floor level a function is placed. In the showcase the advantage of using 3D data properly is demonstrated by designing the building according to the sound pressure levels that are available from the environmental data in the 3D spatial plan (see Figure 8).
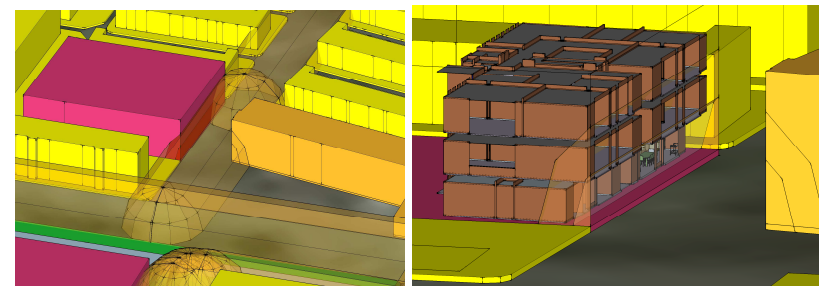

Figure 8. Sound pressure levels in 3D (left) and a building designed accordingly (right)

Step 4c: The architect checks the design for regulations regarding cultural heritage in the underground

If a spatial object has cultural or archaeological value, this restricts the possibilities for building on the entire location. At times however, it may be known that only part of a building plot has a chance of containing artefacts or remains of ancient structures. Or perhaps they are known to only be found beyond a certain depth. If this is the case, it makes sense to model this in $3 \mathrm{D}$, thereby increasing the usability of the land. In this showcase an object (a white box) has been modelled below the surface, that has been taken into account in the structural design of the foundation (see Figure 9).

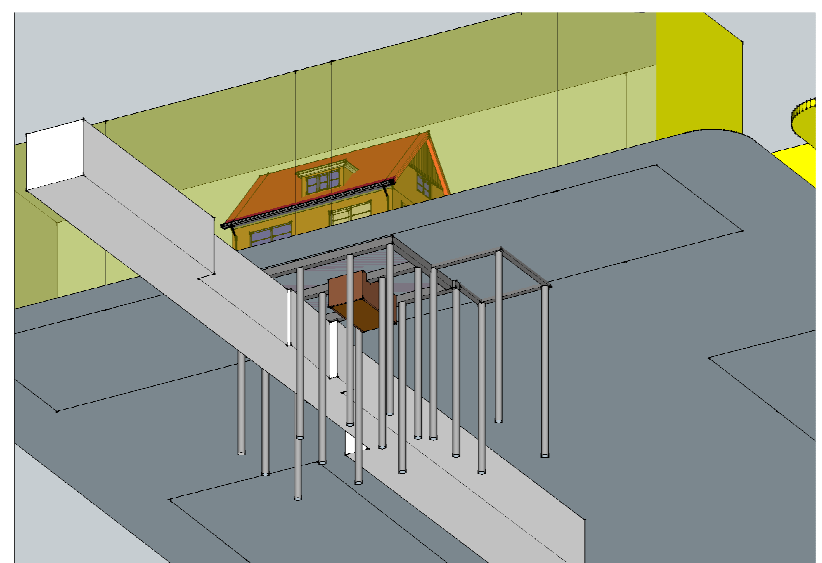

Figure 9. An object below the surface with archaeological value has been taken into account in the structural design

Step 5: The architect uploads the IFC model to the building permit portal

The final step in the workflow is uploading the final design as an IFC model to the building permit portal. The municipality will check whether the design indeed complies with the regulations, but the process will be significantly shorter, since much of the checking is automated and they use the same data as the designer.

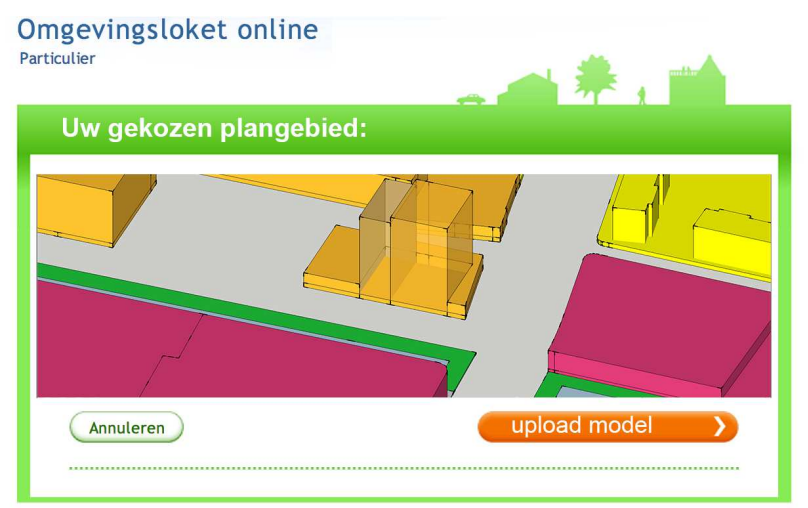

Figure 10. Mock-up of the building permit portal for 3D models

\subsection{Remarks}

The presented workflow is realistic, but it is not yet reality. Due to constraints in time and budget some concessions had to be made in the development of the showcase. The choice was made to elaborate a workflow that shows multiple possibilities of the technology instead of fine tuning a single piece of the workflow or elaborating one specific design.

Also, due to the given limitations not all possible environmental data was incorporated in the showcase. In practice, the number of sub steps of step 4 (checking) may increase according to the complexity of the building location. More environmental constraints will lead to more data to be considered by the designer and to be checked by the software. In addition, step 3 (design) and step 4 (checking) are likely to form an iterative process until the final design is established.

\section{FINDINGS}

The experience in this project has shown that the integration of $3 \mathrm{D}$ spatial plans and BIM is beneficial in the design process and offers significant innovations. Technically, it seems possible to establish the architecture described in the showcase. Although, there are a few issues that need to be resolved before the architecture can become widely used practice. Most of the issues relate to standardisation and agreements about data modelling. On top of that, there are other issues regarding 3D spatial planning and BIM that require consideration as well.

\subsection{Modelling issues}

Before spatial planning and BIM can be integrated in 3D, the (national) standard for spatial planning needs to be extended with a $3 \mathrm{D}$ component. This is not straightforward. In 2D, the spatial planning map is a planar partition. Should this be the case in 3D (i.e. every space should get a destination)? And how closely related should the $3 \mathrm{D}$ objects be with the $2 \mathrm{D}$ planning objects, i.e. should they always relate to a planning object at ground surface or is it possible that one 3D planning object (for example for the underground storage of heat/cold) crosses several 2D planning objects. Another issue is to define the reference level. In the verbal regulations that accompany the spatial planning data, the reference level is defined as the point on the road, which is closest to the design area. In 3D one should work with real world coordinates defined in the national reference system (for $\mathrm{x}, \mathrm{y}$ this is already covered in the workflow described in the showcase).Apart from these issues related to spatial plans, some other technical issues require further 
attention. The first is how to respect the differences between accuracy and precision in the BIM data on the one hand and the environmental data on the other hand. BIM models are mostly modelled with millimetre accuracy, whereas geo-information is nowhere near that accuracy (and is not intended to be). This is specifically relevant in the analysis that checks whether the designed building is valid according to the regulations for geometry. On this issue it is important to consider that this 'error margin' is existent in current practice as well. Automated checking just makes the issue more explicit, compared to manual checking.

In IMRO regulations can be described in text. For automated checking, the model needs standardised attributes. The process towards automated checking therefore requires commitment and willingness of local governments to adopt the new technologies. Both on the 3D IMRO modelling side, as on the IFC modelling side the attributes need to be standardized to allow efficient automated checking.

\subsection{Technical issues}

On the technical side, the transformation from 3D IMRO GML (which is a GML/CityGML based model) to IFC (and vice versa) needs to be improved. At this moment most of the known tools do not deliver a reliable result after transformation. This is crucial to allow automated transformation from 3D IMRO to the IFC model used by architects in the design process. The work of Benner, Geiger, and Häfele (2010), van Berlo and de Laat (2011) and Isikdag and Zlatanova (2009), addresses most of the issues we also found during the development of this showcase.

On the BIM side it is a known issue that import functions from some of the large BIM modelling software tools are not always reliable. Some BIM modelling software won't import all of the IFC data. This issue is well known in the AEC industry and a large effort is being made to resolve these issues.

The proposed showcase in this project relies heavily on the use of WebGL technology to visualise 3D models in a web browser. WebGL implementations in several web browsers are not always stable enough to work in a legal environment like a National Spatial planning portal. It is expected that some browsers will have stable implementations on a short term, but a browser check would still be needed to check compatibility.

\section{CONCLUSION}

In the presented showcase the geo-information was imported into the BIM environment. The advantage is that clashdetection and rule checking, needed for the spatial analyses, is standard functionality in BIM software in contrast to GIS software. The disadvantage is that in this case the data is copied without retaining a reference to the source. This introduces a risk of inconsistencies.

In the Netherlands, the (quality of) spatial plans are the responsibility of the municipalities. They will need to comply with standards that are enforced on a national level, in the current situation however, these standards do not force regulations to be encoded as attributes. Regulations described in text are also allowed. For automated checking, attributes are highly preferable over text and the process towards automated checking therefore requires commitment and willingness of local governments to adopt the new technologies. This will also require an investment on their part to build in house knowledge and experience with the new technologies. This process should go hand in hand with the development of standards for 3D spatial planning and incorporation of spatial planning attributes in BIM standards.

Finally, commitment of the national government is required to arrange the legal boundaries for automated checking of building permits. Currently, spatial and environmental planning policy are being reformed by the government in a programme named 'simply/simple better' (Dutch: Eenvoudig Beter). We are confident that the 3D technology is at the required level. If the stakeholders involved can make the necessary arrangements integration of 3D spatial planning and BIM fits right in.

\section{INTERNATIONAL RECOMMENDATIONS}

Many research activities on the integration between BIM and GIS has a focus on getting BIM data into the GIS context. BIM is seen as a source for Geospatial oriented data stores. In the presented showcase the geo-information was imported into a BIM environment. This experiment has shown that BIM and GIS are still two separate worlds with their own processes, standards and culture. The geospatial data that is available is of better use to architects, designers and engineers when it is available as a BIM model. Standard functionality in BIM software can be used and people feel comfortable using their own tools and methodologies. First responses from users in the field indicate that they think work can be much more effective and efficient because they can work within their own comfort zone. A conclusion of this experiment might be that integration of BIM and GIS or flawless data exchange between BIM and GIS is mostly a scientific challenge Professionals in the construction industry can make better use of regulations and spatial data if they are available in their familiar BIM design environment. To what extent this BIM information is converted from or exchanged with GIS is less relevant for them. More research is required to validate this hypotheses.

Even if this is the case, there still needs to be awareness and understanding of the differences between geo-information and building information. When geo-information is used as starting data for a design, architects and designers need some understanding of the lower precision levels that are in the nature of geo-information. Technical solutions may help to get the data from one data standard to another, but arrangements will have to be made between the parties that are modelling and checking in both geo-information and building information.

Arrangements will also need to be made by the parties that perform measurements in the environment. In current practice, measurements on noise, pollution, archaeology etc. result in elaborate annexes to the spatial plan. On the road to automated checking, measurement data on a wide array of environmental aspects should be stored uniformly and in such a way that it can be related to a 3D spatial model, in BIM and/or GIS. If it can also be visualized, this will be a great help to designers, civil servants and civilians and it is likely to prevent many misunderstandings in the building permit process.

\section{REFERENCES}

Benner, Joachim, Andreas Geiger, and Karl-Heinz Häfele. 2010. "Concept for Building Licensing Based on Standardized 3d Geo Information." In Proc. of the 5th International $3 D \quad$ GeoInfo Conference. http://www.3dgeoinfo.org/ISPRS_Conference_CD/Pa per_ISPRS/Oral/2_3DGeoInfo2010_105_Benner_Bui ldingLicensing.pdf. 
ISPRS Annals of the Photogrammetry, Remote Sensing and Spatial Information Sciences, Volume II-2/W1, ISPRS 8th 3DGeolnfo Conference \& WG II/2 Workshop, 27 - 29 November 2013, Istanbul, Turkey

Bormann. 2010. "From GIS to BIM and Back again-A Spatial Query Language for 3D Building Models and 3D City Models." In . International Archives of the Photogrammetry, Remote Sensing and Spatial Information Sciences, Volume XXXVIII-4/W15.

Berlo, Léon van and Ruben de Laat. 2011. "Integration of BIM and GIS: The Development of the CityGML GeoBIM Extension." In Advances in $3 D$ Geo-Information Sciences, 211-225. Springer. http://link.springer.com/chapter/10.1007/978-3-64212670-3_13.

El-Mekawy, Mohamed, Anders Östman, and Khurram Shahzad. 2011. "Towards Interoperating CityGML and IFC Building Models: A Unified Model Based Approach." In Advances in 3D Geo-Information Sciences, edited by Thomas H. Kolbe, Gerhard König, and Claus Nagel, 73-93. Lecture Notes in Geoinformation and Cartography. Springer Berlin Heidelberg. http://link.springer.com/chapter/10.1007/978-3-64212670-3_5.

Isikdag, Umit, and Sisi Zlatanova. 2009. “Towards Defining a Framework for Automatic Generation of Buildings in CityGML Using Building Information Models." In 3D Geo-Information Sciences, edited by Jiyeong Lee and Sisi Zlatanova, 79-96. Lecture Notes in Geoinformation and Cartography. Springer Berlin Heidelberg.

http://link.springer.com/chapter/10.1007/978-3-54087395-2_6. 